\title{
Cytotoxic effect of Artesunate on myeloid leukemia cell lines through up-regulating miR-29c expression
}

\author{
Cui-Yun Dou ${ }^{1 \#}$, Lu-Lu Liu ${ }^{2 \#}$, Feng Li ${ }^{3 \#}$, Lei Liu ${ }^{1}$, Cui-Ling Wang ${ }^{1}$, Li-Na Wang ${ }^{2}$, Hai-Hua Wang ${ }^{2}$, \\ Hao Zhang ${ }^{1}$, Ming-Tai Chen ${ }^{2}$ \\ ${ }^{1}$ Department of Hematology, ${ }^{2}$ Central Laboratory, Affiliated Hospital of Jining Medical University, Jining 272029, China; ${ }^{3}$ Department of Molecular \\ Biology, Shanxi Cancer Hospital, Affiliated Cancer Hospital of Shanxi Medical University, Taiyuan 030012, China \\ \#These authors contributed equally to this work. \\ Correspondence to: Prof. Hao Zhang; Dr. Ming-Tai Chen. Affiliated Hospital of Jining Medical University, 89 Guhuai Road, Jining 272029 , China. \\ Email: gx-zhanghao@126.com; chenmt.sdubio@163.com.
}

Submitted Aug 17, 2018. Accepted for publication Nov 21, 2018.

doi: $10.21037 /$ tcr.2018.11.36

View this article at: http://dx.doi.org/10.21037/tcr.2018.11.36

Acute myeloid leukemia (AML) is a malignant hematopoietic neoplasm characterized by arrest of myeloid differentiation, rapid growth and apoptotic repression of leukemic blasts that arise from the hematopoietic stem/progenitor cell (HSPC) population within the bone marrow (BM) (1). In the past three decades, great progress has been made in understanding AML pathogenesis and clinical treatment with allogeneic stem cell transplantation (2). However, the current AML therapeutic regimen only cures $20 \%$ of the patients (3), highlighting the urgent necessity for discovery and development of innovative agents and novel therapeutic strategies to improve present situation of AML treatment.

Artesunate (ART), a semi-synthetic derivative of artemisinin, is one of the most commonly used anti-malarial drugs. Nowadays, ART has been reported to exert antitumor potential by inducing cell apoptosis and inhibiting cell growth in many cancers (4). The anti-leukemic property of ART was also demonstrated in leukemia cell lines (5). However, the exact role and mechanism of ART in AML remain to be determined. Thus in this study, we used $50 \mu \mathrm{M}$ ART to treat two myeloid leukemia cell lines, HL-60 and THP-1, for 48 hours. As one of the cytoskeletal actin, betaactin is involved in the cell motility, structure and integrity, whose immunofluorescence staining was performed here to evaluate the cell morphological changes. As shown in Figure $1 A, B$, ART treatment resulted in more twisted and broken HL60 and THP-1 cells with fragmented nuclei (DAPI staining) compared to the untreated group, indicating the cytotoxic effect of ART on HL-60 and THP-1 leukemia cells. Next, we further investigate the influence of ART on leukemia cell proliferation using carboxyfluorescein diacetate succinimidyl ester (CFSE) cell division assay kit. CFSE is a fluorescent dye which can track the cell division. HL-60 and THP-1 cells were first labeled with CFSE using its work solution for 30 minutes according to the manufacturer's instruction in a $37^{\circ} \mathrm{C}$ incubator with $5 \% \mathrm{CO}_{2}$, then rinsed twice with PBS, followed by culture in medium with and without $50 \mu \mathrm{M}$ ART for 48 hours. Next, the cells were collected for flow cytometric analysis. The peak in the flow chart gradually shifts to the left with the cells dividing. As shown in Figure 1C,D, the division of HL-60 and THP-1 cells was remarkably inhibited by $50 \mu \mathrm{M}$ ART treatment with the peak in the right of that representing CFSE-labeled but ARTuntreated cells. To reveal the molecular mechanism underlying the cytotoxic effect of ART on myeloid leukemia cells, HL-60 and THP- 1 cells were cultured in medium with and without 25 and $50 \mu \mathrm{M}$ ART for 48 hours. Then the cells were collected for RNA extraction and the mRNA expression of cell apoptosisand proliferation-associated genes (Bcl2, Mcl1, c-Myc, CDK6 and CCND2) were detected. The results showed that the antiapoptotic gene $B c l 2$ and cell cycle associated gene CCND2 exhibited decreased expression in both HL-60 and THP-1 cells in ART-dose-dependent manner (Figure 1E,F,G,H), but the same results were not observed for Mcl1, c-Myc and CDK6. The down-regulation of $\mathrm{Bcl} 2$ and $\mathrm{CCND} 2$ to some extent accounted for the cytotoxic effect and division inhibition of ART on leukemia cells. On the other hand, we verified that ART has little influence on myeloid differentiation of THP-1 and HL-60 cells induced by phorbol 12-myristate 13-acetate (PMA) and alltrans retinoic acid (ATRA) respectively in vitro (data not shown).

MicroRNAs (miRNAs) are a class of 21-23 nt small 

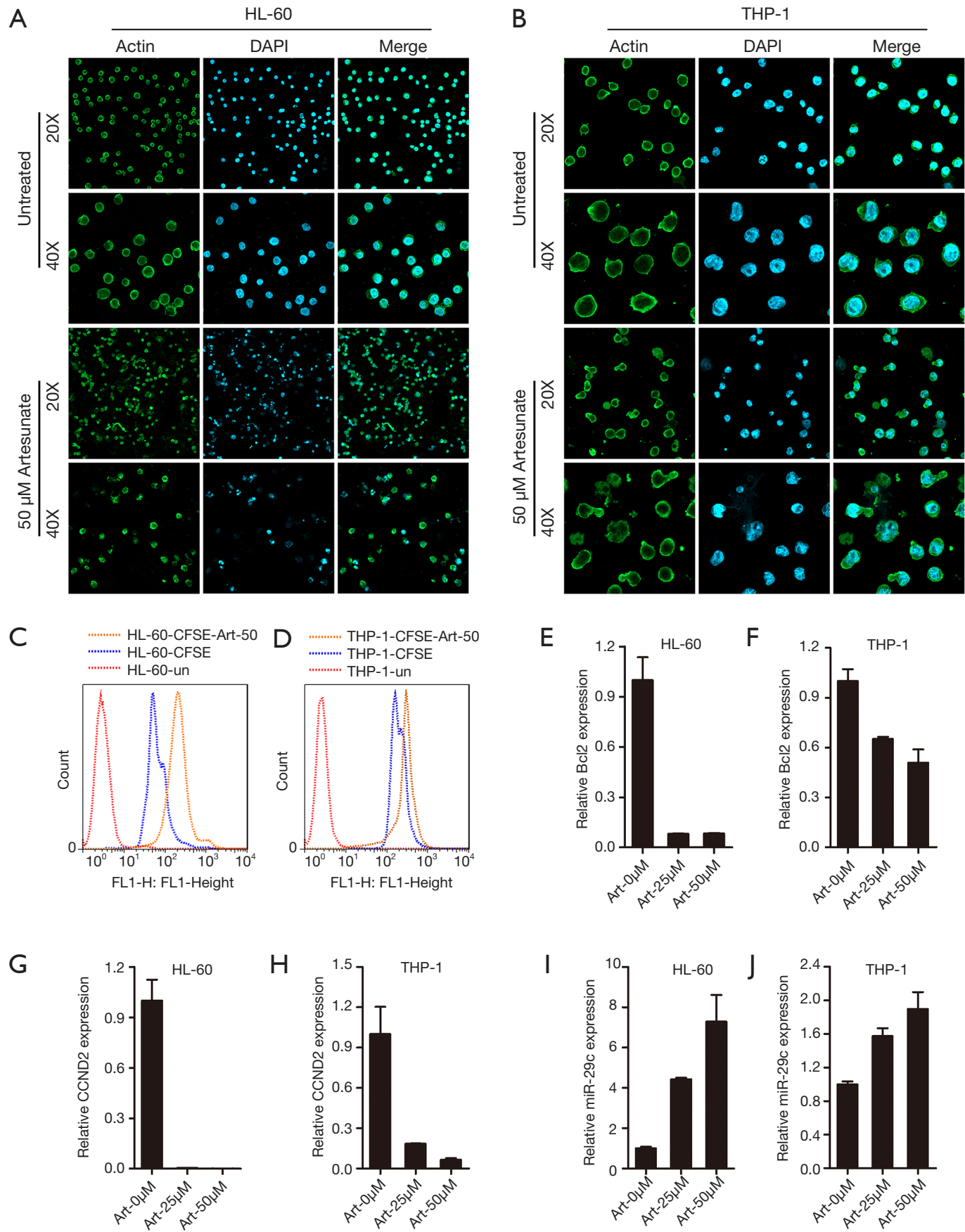

Figure 1 Cytotoxic effect of artesunate on HL-60 and THP-1 leukemia cells. (A,B) HL-60 (A) and THP-1 (B) leukemia cells were cultured with and without $50 \mu \mathrm{M}$ ART for 48 hours. Then the cells were collected for beta-actin immunofluorescence staining and the pictures were captured using ZEISS LSM 800 confocal microscope under 20× and 40x objective magnification. (C,D) HL-60 (C) and THP-1 (D) cells were pre-labeled with CFSE according to the manufacturer's instruction, followed by culture in medium with and without $50 \mu \mathrm{M}$ ART for 48 hours. Then the cells were collected for flow cytometric analysis. (E,F,G,H,I,J) HL-60 and THP-1 cells were cultured with and without $25 \mu \mathrm{M}$ and $50 \mu \mathrm{M}$ ART for 48 hours. (E,F) Bcl2 mRNA expression was detected using qRT-PCR in ART-treated HL-60 (E) and THP-1 (F) cells. GAPDH was used as a loading control. (G,H) CCND2 mRNA expression was detected using qRT-PCR in ART-treated HL-60 (G) and THP-1 (H) cells. GAPDH was used as a loading control. (I,J) MiR-29c expression was detected using qRT-PCR in ART-treated HL-60 (I) and THP-1 (J) cells. U6 snRNA was used as a loading control. ART, artesunate; CFSE, carboxyfluorescein diacetate succinimidyl ester. 
non-coding RNAs and can post-transcriptionally regulate gene expression by binding to the 3 '-untranslated region (3'-UTR) of target mRNAs through their seed sequences, which have been identified as crucial regulators in normal and malignant biological processes (6). To investigate whether miRNAs mediate the cytotoxic effect of ART on leukemia cells, we screened a series of miRNAs and detected their expression in ART-treated HL-60 and THP-1 cells. MiR-29c was found to exhibit increased expression in ARTdose-dependent manner (Figure 1I,7). MiR-29c is one of the miR-29 family members that have been intensively studied and function as classical tumor suppressors in mantle cell lymphoma, gastric cancer and leukemia (7) via promoting cell apoptosis and repressing cell proliferation. Besides, the up-regulation of miR-29c resulted from ART treatment also explained the decreased expression of CCND2 which has been validated as a down-stream target of miR-29c (8).

In summary, our results demonstrated that ART exhibited obvious cytotoxic effect on HL-60 and THP-1 myeloid leukemia cell lines, which was presented as broken cell morphology and repressed cell division. Preliminary mechanistic analysis revealed that ART may exert its anti-leukemic property by increasing miR-29c expression. Though it still remains further elucidated about the cytotoxic effect of ART on primary AML cells, whether miR-29c mediates the cytotoxic effect of ART by targeting $\mathrm{Bcl} 2$ and CCND2 expression in leukemia cells and the molecular mechanism of increased miR-29c expression elicited by ART administration, our finding may provide a novel promising therapeutic strategy for AML using combined treatment of ART plus other agents or miR-29c manipulation.

\section{Acknowledgments}

Funding: This work was supported by the National Natural Science Foundation of China (81700099), the Natural Science Foundation of Shandong Province, China (ZR2015HL007 and ZR2017LH029), the Health and Family Planning Commission of Shandong Province, China (2016WS0182) and Supporting Fund for Teachers' research of Jining Medical University (JYFC2018FKJ112).

\section{Footnote}

Provenance and Peer Review: This article was a standard submission to the journal. The article has undergone external peer review.

Conflicts of Interest: All authors have completed the ICMJE uniform disclosure form (available at http://dx.doi. org/10.21037/tcr.2018.11.36). The authors have no conflicts of interest to declare.

Ethical Statement: The authors are accountable for all aspects of the work in ensuring that questions related to the accuracy or integrity of any part of the work are appropriately investigated and resolved.

Open Access Statement: This is an Open Access article distributed in accordance with the Creative Commons Attribution-NonCommercial-NoDerivs 4.0 International License (CC BY-NC-ND 4.0), which permits the noncommercial replication and distribution of the article with the strict proviso that no changes or edits are made and the original work is properly cited (including links to both the formal publication through the relevant DOI and the license). See: https://creativecommons.org/licenses/by-nc-nd/4.0/.

\section{References}

1. Dohner H, Weisdorf DJ, Bloomfield CD. Acute Myeloid Leukemia. N Engl J Med 2015;373:1136-52.

2. Atlas MP, Lipton JM. Treatment of relapsing AML following hematopoietic stem cell transplantation. Pediatr Transplant 2003;7:419-21.

3. Tallman MS, Gilliland DG, Rowe JM. Drug therapy for acute myeloid leukemia. Blood 2005;106:1154-63.

4. Slezakova S, Ruda-Kucerova J. Anticancer Activity of Artemisinin and its Derivatives. Anticancer Res 2017;37:5995-6003.

5. Li Y, Feng L, Jiang W, et al. Artesunate possesses antileukemia properties that can be enhanced by arsenic trioxide. Leuk Lymphoma 2014;55:1366-72.

6. Bartel DP. MicroRNAs: genomics, biogenesis, mechanism, and function. Cell 2004;116:281-97.

7. Schmitt MJ, Margue C, Behrmann I, et al. MiRNA-29: a microRNA family with tumor-suppressing and immunemodulating properties. Curr Mol Med 2013;13:572-85.

8. Gong JN, Yu J, Lin HS, et al. The role, mechanism and potentially therapeutic application of microRNA-29 family in acute myeloid leukemia. Cell Death Differ 2014;21:100-12.

Cite this article as: Dou CY, Liu LL, Li F, Liu L, Wang CL, Wang LN, Wang HH, Zhang H, Chen MT. Cytotoxic effect of Artesunate on myeloid leukemia cell lines through up-regulating miR-29c expression. Transl Cancer Res 2018;7(6):1748-1750. doi: $10.21037 /$ tcr.2018.11.36 